\title{
Spectrum Sharing and Energy-Efficient Power Optimization for Two-tier Femtocell Networks
}

\author{
Ishtiaq Ahmad*, Zhiyong Feng ${ }^{\dagger}$, Atif Hameed *, Ping Zhang ${ }^{\dagger}$ and Yichen Zhao ${ }^{\dagger}$ \\ Key Laboratory of Universal Wireless Communications, Ministry of Education \\ Wireless Technology Innovation Institute (WTI), Beijing University of Posts and Telecommunications \\ Beijing, P.R.China, 100876 \\ email:* ahmedishtiaq1971, atif95\}@gmail.com, ${ }^{\dagger}\{$ fengzy, pzhang, zyc891013\} @bupt.edu.cn
}

\begin{abstract}
Two-tier femtocell network is considered a promising solution for the rapidly rising mobile data rate demand due to its high spectrum efficiency. However, underlaying femtocells with macrocells will result in co-channel interference (CCI) which severely deteriorates network performance. Therefore, optimized power control is required in femtocell network to avoid such interference. Additionally, energy efficiency of cellular network is becoming increasingly important under current trend of green communication. Thus, in this paper, we propose an energy efficient power control algorithm for femtocell network. Our proposed model is based on Stackelberg game, in which macro base station (MBS) acts as leader while femto base stations (FBSs) act as followers. MBS adjusts its power and imposes interference price on femtocells to maintain its user's minimum rate requirement and earns revenue. Subsequently, FBSs optimize their power based on the imposed price which takes into account the cost of both spectrum sharing and energy usage. Two cases, namely uniform and non-uniform pricing are considered. And an iterative algorithm which converges rapidly is also proposed to calculate the interference price and power allocation strategy in the two cases. Finally, our model is validated by simulations and the results show the superior performance of our schemes over existing one.
\end{abstract}

Index Terms-Femtocells, interference, energy efficiency, Stackelberg game, convex optimization.

\section{INTRODUCTION}

Exponential increase in wireless data rate demand urges us to look for new paradigms, such as more aggressive frequency reuse and smaller cell size. And low power and low cost short range femtocell is proposed as a promising solution. It provides high signal strength and improves indoor coverage. However, when underlaying femtocells with macrocell, some technical issues need to be resolved such as co-tier and cross-tier interferences, which severely impair the network performance. The interference can be more serious when spectrum sharing rather than spectrum splitting is adopted. Therefore, power control for interference management in twotier femtocell network is proposed, which has been frequently discussed in previous literature [1]-[4].

On the other hand, current high data rate applications also increases communication power consumption which consequently raises power cost [5]. Thus energy efficiency (EE) is becoming increasingly important in addition to spectrum efficiency and green communication is widely studied now-adays. Therefore, the energy aspect needs to be considered for power control in femtocell networks. In [6], energy efficient power optimization for heterogeneous network with femtocells and cognitive radios is studied. The problem is formulated via game theory, and backward induction is used to solve the game and prove the Stackelberg game equilibrium (SE). Similarly, energy efficient power optimization for the uplink of two-tier femtocell network is addressed in [7] using fictitious game, in which each user announces interference price to other users to compensate the interference caused by others. In [8], maximum outage capacity in dense indoor femtocell network is studied with joint energy and spectrum utilization. In [9] energy efficient power optimization is modeled based on non-cooperative game with dual utility, where each MBS maximizes its utility considering both circuit and transmission power while each FBS improves its SINR utility.

In this paper, we also present an energy efficient power optimization scheme for spectrum sharing two-tier macrofemto network. We model the power control problem using Stackelberg game, where macro base station (MBS) works as leader and femtocells work as followers. In contrast to [4] and [10], dynamic power allocation is adopted at MBS to adjust its interference tolerance for earning more revenue and maintaining its user's quality of service (QoS) while MBS interference tolerance is assumed fixed in [4] and [10]. The interference price is calculated by considering both the spectrum sharing cost and energy usage by the FBSs. We analyze two cases of interference pricing i.e. uniform and non-uniform pricing and propose a fast converging iterative algorithm to calculate the effect of co-tier interference on price determination while it is assumed fixed in [4] and [10]. Simulation results show the enhanced performance of our proposed schemes over existing one.

The rest of the paper is organized as follows. In Section II, we describe the system model and formulate the problem in Section III based on Stackelberg game. Section IV and Section $\mathrm{V}$ describe energy efficient pricing and power allocation based on non-uniform and uniform pricing respectively. The simulation results are presented in Section VI. Finally, Section VII concludes the paper.

\section{Network Model}

We consider a two-tier femtocell network, which consists of one MBS and $N$ spectrum sharing femtocells. MBS and 
each femtocell schedule on user for a specific sub-channel i.e., orthogonal transmission is assumed. Each base station and user is equipped with single antenna. We consider the downlink scenario, in which a MUE near the femto network is being interfered by their downlink transmission. Let $h_{i i}$ and $h_{m m}$ denote the channel gains respectively, from $i^{\text {th }}$ FBS and MBS to their scheduled users. Similarly, $g_{i m}$ and $g_{m i}$ are the cross channel gains from $i^{t h}$ FBS and MBS to the users in opposite cells. If $p_{i}$ is the transmitting power of $i^{t h}$ FBS and $p_{m}$ is the transmitting power of MBS then their signal-to-interferenceplus-noise ratios $\gamma_{i}$ and $\gamma_{m}$ respectively, can be written as

$$
\begin{gathered}
\gamma_{i}=\frac{p_{i} h_{i i}}{\sum_{j \neq i} p_{j} h_{i j}+p_{m} g_{m i}+\sigma_{i}^{2}} \quad \forall i . \\
\gamma_{m}=\frac{p_{m} h_{m m}}{\sum_{i} p_{i} g_{i m}+\sigma_{m}^{2}},
\end{gathered}
$$

where $\sigma^{2}$ is the background noise. The term $\sum_{j \neq i} p_{j} h_{i j}$ in (1) is the inter-femtocells interference and $p_{m} g_{m i}$, represents interference from MBS to the $i^{\text {th }} \mathrm{FUE}$ while $\sum_{i} p_{i} g_{i m}$ in (2) is integrated interference from the femtocell network to MUE. An interference management scheme should ensure that integrated interference from the femtocell network is under the interference tolerance level of MUE. Mathematically, we can write

$$
\sum_{i} p_{i} g_{i m} \leq Q
$$

where $Q$ is the interference tolerance of the MUE.

\section{PROBLEM Formulation}

To control the interference from femtocell network, we formulate bi-level Stackelberg game, where MBS acts as a leader and imposes interference price to reap revenue from the femtocell network by selling out its interference tolerance as represented by (3). The FBSs act as followers and calculate their power allocation based on imposed price non-cooperatively. Let $\Lambda=\left[\lambda_{1}, \lambda_{2}, \ldots \ldots, \lambda_{N}\right]$ and $\mathrm{P}=\left[p_{1}, p_{2}, \ldots \ldots, p_{N}\right]$ denote the femtocells interference price and power allocation vectors, respectively. The EE performance can be evaluated by taking power consumption into account as defined in [6], thus the leader's sub-game or upper-sub game can be written as

Problem 3.1 (Upper sub-game)

$$
\begin{gathered}
\max _{p_{m}>0, \Lambda>0} U_{m}\left(\Lambda, \mathrm{P}, p_{m}\right)=\sum_{i} \lambda_{i} p_{i} g_{i m}-u_{m} p_{m}, \\
\text { s.t. } \log \left(1+\gamma_{m}\right) \geq R^{\mathrm{min}} .
\end{gathered}
$$

where $R^{\text {min }}$ is the minimum rate requirement of MUE which acts as an indicator of QoS. Based on (5), we can rewrite (3) as

$$
\sum_{i} p_{i} g_{i m} \leq \frac{p_{m} h_{m m}}{e^{R^{\min }}-1}-\sigma_{m}^{2}
$$

The objective function in (4) consists of two parts i.e., profit and cost. The term $\sum_{i} \lambda p_{i} g_{i m}$ represents profit i.e. revenue earned by the MBS. While $u_{m} p_{m}$ denotes power cost of the MBS, where $u_{m}$ is cost factor for unit power. By increasing its power, MBS can increase its interference tolerance, which will subsequently increase its revenue. However, increasing power will also increase its cost. Therefore, by adding power consumption in the objective function, our algorithm can avoid rising transmitting power blindly to earn more revenue. Thus, the energy efficiency can be optimized with power allocation and the tradeoff between revenue and energy consumption can be adjusted by the cost of unit energy. The MBS revenue also depends upon interference price $\Lambda$. If MBS increases its price to earn more revenue, the FBSs will buy less interference quota due to high cost, which will consequently, decreases MBS revenue. So, we need to determine the optimal interference prices that maximize MBS utility.

Similarly the followers sub-game or lower sub-game can be expressed as

Problem 3.2 (Lower sub-game):

$$
\max _{p_{i}>0} U_{i}\left(p_{i}, \lambda_{i}\right)=\log \left(1+\gamma_{i}\right)-\lambda_{i} p_{i} g_{i m}-u_{i} p_{i} .
$$

The objective function (7) has three terms i.e. one profit and two cost terms respectively. The first term is the transmission rate and the second term denotes the interference cost paid by $i^{t h}$ FBS while the third term represents energy cost, where $u_{i}$ denotes per unit power cost. Through increasing its power, FBSs can increase its transmission rate. However, increasing power also means higher energy cost and more interference to nearby MUE, which will decrease the revenue. This equilibrium can prevent the FBSs to increase their power in a greedy manner. Similarly, we also need to determine the optimal power of FBSs which can maximize their utility functions.

The game is organized in such way that players in both upper and lower sub-games can jointly maximize their utility functions. The Stackelberg game solution can be found through Nash Equilibrium, where neither leader nor followers have incentive to deviate from their strategy unilaterally. Let denote $\Lambda^{*}$ and $\mathrm{P}^{*}$ as the optimal price and the optimal power vectors respectively, the following condition must be satisfied.

$$
\begin{gathered}
U_{m}\left(\Lambda^{*}, \mathrm{P}^{*}\right) \geqslant U_{m}\left(\Lambda, \mathrm{P}^{*}\right) . \\
U_{i}\left(p_{i}^{*}, \lambda_{i}^{*}\right) \geqslant U_{i}\left(p_{i}, \lambda_{i}^{*}\right) \forall i .
\end{gathered}
$$

\section{EnERgy EFFicient Power Allocation With NON-UNIFORM PRICING}

When non-uniform pricing strategy is adopted, the MBS sets different price $\lambda_{i}$ for each FBS based on its induced interference. In this game the leader moves first and determines the interference price while the followers observe the interference price and determine their transmitting power accordingly. Then the leader calculates its best response based on the strategies adopted by the followers. The game can be carried out in an iterative manner that reaches to the optimal solution in the end. For a given $\lambda_{i}$, first we calculate the optimal power $p_{i}^{*}$ for the followers by solving Problem 3.2. Since the utility function given by (7) is concave, there exists at least one Nash equilibrium point which can be calculated using typical water filling 


$$
\begin{gathered}
p_{i}^{*}\left(\lambda_{i}, p_{m}\right)=\left[\frac{1}{\lambda_{i} g_{i m}+u_{i}}-\frac{p_{m} g_{m i}+I_{i}+\sigma_{i}^{2}}{h_{i i}}\right]^{+} . \\
\text {where } I_{i}=\sum_{j \neq i} p_{j} h_{i j} .
\end{gathered}
$$

The solution given by (10) is the optimal strategy of femtocell $i$ if the strategy of other femtocells is known. From (10), we know that transmitting power has to be positive, thus the $i^{\text {th }}$ FBS can only transmit if (12) is satisfied, otherwise it will be removed from the game.

$$
\lambda_{i}<\frac{h_{i i}}{g_{i m}\left(p_{m} g_{m i}+I_{i}+\sigma_{i}^{2}\right)}-\frac{u_{i}}{g_{i m}} .
$$

To determine the solution of the upper sub-game, let first consider the rate requirement of MUE, which is shown in (5). The optimized power of MBS can be written as

$$
p_{m}^{*}(\mathrm{P})=\frac{\left(e^{R^{\mathrm{min}}}-1\right)\left(\sum_{i} p_{i} g_{i m}+\sigma_{m}^{2}\right)}{h_{m m}} .
$$

Replacing the value of $p_{i}$ in (13), we can rewrite the expression as

$$
\begin{gathered}
p_{m}(\Lambda)=R \sum_{i}\left(\frac{g_{i m}}{\lambda_{i} g_{i m}+u_{i}}-\frac{g_{i m}\left(I_{0}+\sigma_{i}^{2}\right)}{h_{i i}}\right)+R \sigma_{m}^{2}, \\
\text { where } R=\frac{\left(e^{R^{\mathrm{min}}}-1\right)}{h_{m m}+\left(e^{R^{\mathrm{min}}}-1\right) \sum_{i} \frac{g_{i m} g_{m i}}{h_{i i}}} .
\end{gathered}
$$

Similarly, from (10) and (14) we rewrite $i^{\text {th }}$ FBS power as function of $\Lambda$ as

$$
\begin{gathered}
p_{i}(\Lambda)=\left[\frac{1}{\lambda_{i} g_{i m}+u_{i}}-\frac{I_{0}+\sigma_{i}^{2}}{h_{i i}}-\frac{g_{m i} R}{h_{i i}} \sigma_{m}^{2}\right. \\
\left.-\frac{g_{m i} R}{h_{i i}} \sum_{i}\left(\frac{g_{i m}}{\lambda_{i} g_{i m}+u_{i}}-\frac{g_{i m}\left(I_{0}+\sigma_{i}^{2}\right)}{h_{i i}}\right)\right] .
\end{gathered}
$$

Again, replacing the value of $p_{i}^{*}$ and $p_{m}^{*}$ in objective function (4), we can re-formulate it as a function of interference price only, which can be expressed as:

Problem 4.1:

$$
\begin{gathered}
\max _{\lambda_{i}>0} U_{m}(\Lambda)=\sum_{i}\left[\frac{\lambda_{i} g_{i m}}{\lambda_{i} g_{i m}+u_{i}}-\frac{\lambda_{i} g_{i m}\left(I_{0}+\sigma_{i}^{2}\right)}{h_{i i}}-\right. \\
\left.\frac{\lambda_{i} g_{i m} g_{m i}}{h_{i i}} R\left(\sum_{i}\left(\frac{g_{i m}}{\lambda_{i} g_{i m}+u_{i}}-\frac{g_{i m}\left(I_{0}+\sigma_{i}^{2}\right)}{h_{i i}}\right)+\sigma_{m}^{2}\right)\right] \\
-u_{m} R\left(\sum_{i} \frac{\lambda_{i} g_{i m}}{\lambda_{i} g_{i m}+u_{i}}-\sum_{i} \frac{g_{i m}\left(I_{0}+\sigma_{i}^{2}\right)}{h_{i i}}+\sigma_{m}^{2}\right) .
\end{gathered}
$$

It is straight forward that the MBS choose the interference price that can maximize its objective function. As (17) is concave, its solution can be expressed as the following proposition.
Proposition 4.1: The optimal solution to the Problem 4.1 can be expressed as

$$
\lambda_{i}^{*}=\sqrt{\frac{\frac{u_{i}}{g_{i m}^{2}}-\frac{g_{m i}}{g_{i m} h_{i i}} R-\frac{u_{m} R}{g_{i m}}}{\frac{I_{0}+\sigma_{i}^{2}}{h_{i i}}+\frac{g_{m i}}{h_{i i}} R\left(\sigma_{m}^{2}-\sum_{i} \frac{g_{i m}\left(I_{0}+\sigma_{i}^{2}\right)}{h_{i i}}\right)}}-\frac{u_{i}}{g_{i m}} .
$$

For proof, the reader is referred to Appendix A.

The solution given by (18) represents the optimal equilibrium interference price. It is observed that interference price is unique thus the SE for the game is unique. We can also determine the optimal power of FBSs and MBS by replacing the value of $\lambda_{i}^{*}$ in (16) and (14) respectively. The FBS for which the interference price does not fulfill the condition (12), its interference price is set to $\infty$ and it will be removed from the game.

Since the co-tier interference at FBSs may change with the power of any other FBS, it is necessary to change the transmitting power to keep up with the new environment. And the new SE point can be reached after sufficient number of iterations. The iterative process proceeds as follows. The central controller (CC) at MBS initializes $p_{m}=0, I_{i}=0$ and calculates the interference price and power for each FBS and MBS using (18) and (16) and (14), respectively. Then, CC determines the value of $I_{i}$ and recalculates the interference price and power. After a few iterations, the interference price and power will converge to its optimal value. Algorithm 1 describes the iterative procedure for price and power allocation.

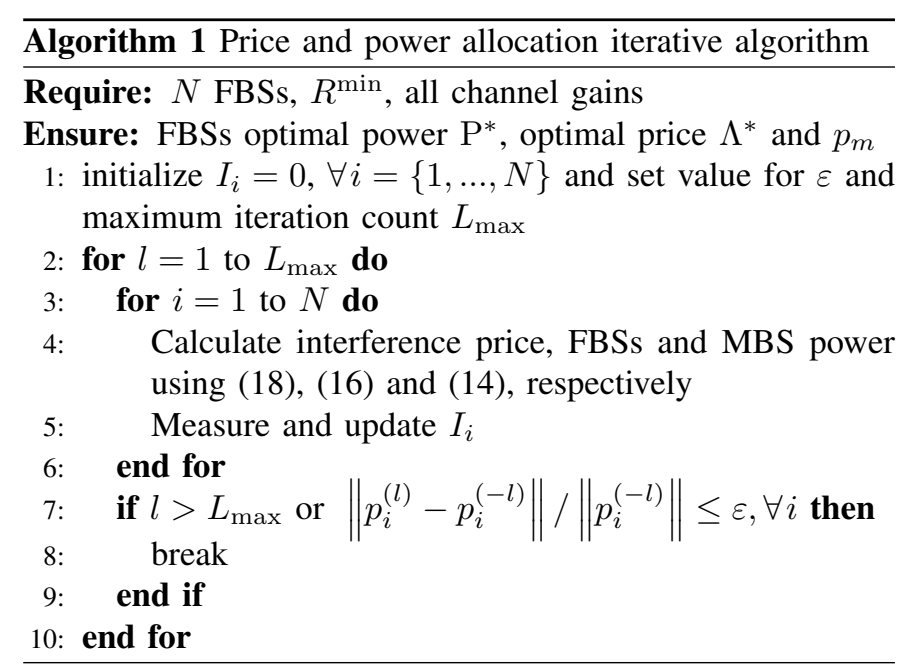

In the above algorithm $L_{\max }$ denotes the maximum number of iterations while $\varepsilon$ denotes error threshold.

\section{Energy EfFicient Power Allocation with UNIFORM PRICING}

Lets assume uniform pricing for all FBS, which means $\lambda_{1}=$ $\lambda_{2}=\ldots \ldots=\lambda_{N}$. Therefore, we denote the interference price as $\lambda$. Then $p_{i}^{*}$ and $p_{m}^{*}$ can be written as (19) and (20), using 
similar methods as given in Section IV.

$$
\begin{gathered}
p_{i}(\lambda)=\left[\frac{1}{\lambda g_{i m}+u_{i}}-\frac{I_{0}+\sigma_{i}^{2}}{h_{i i}}-\frac{g_{m i} R}{h_{i i}} \sigma_{m}^{2}\right. \\
\left.-\frac{g_{m i} R}{h_{i i}} \sum_{i}\left(\frac{g_{i m}}{\lambda g_{i m}+u_{i}}-\frac{g_{i m}\left(I_{0}+\sigma_{i}^{2}\right)}{h_{i i}}\right)\right] \\
p_{m}(\lambda)=R \sum_{i}\left(\frac{g_{i m}}{\lambda g_{i m}+u_{i}}-\frac{g_{i m}\left(I_{0}+\sigma_{i}^{2}\right)}{h_{i i}}\right)+R \sigma_{m}^{2} .
\end{gathered}
$$

Substituting the value of $p_{i}^{*}$ and $p_{m}^{*}$ in objective function (4), we can rewrite it as

Problem 5.1:

$$
\begin{gathered}
\max _{\lambda>0} U_{m}(\lambda)=\sum_{i}\left[\frac{\lambda g_{i m}}{\lambda g_{i m}+u_{i}}-\frac{\lambda g_{i m}\left(I_{0}+\sigma_{i}^{2}\right)}{h_{i i}}-\right. \\
\left.\frac{\lambda g_{i m} g_{m i}}{h_{i i}} R\left(\sum_{i}\left(\frac{g_{i m}}{\lambda g_{i m}+u_{i}}-\frac{g_{i m}\left(I_{0}+\sigma_{i}^{2}\right)}{h_{i i}}\right)+\sigma_{m}^{2}\right)\right] \\
-u_{m} R\left(\sum_{i} \frac{\lambda g_{i m}}{\lambda g_{i m}+u_{i}}-\sum_{i} \frac{\lambda g_{i m}\left(I_{0}+\sigma_{i}^{2}\right)}{h_{i i}}+\sigma_{m}^{2}\right) .
\end{gathered}
$$

The objective function in (21) is concave, thus the optimal price $\lambda$ can be determined by setting $\frac{\partial U_{m}(\lambda)}{\partial \lambda}=0$

Proposition 5.1: The optimal solution to the Problem 5.1 can be written as

$$
\lambda^{*}=\sqrt{\frac{\sum_{i} \frac{u_{i}}{g_{i m}}\left(1-\sum_{i} \frac{g_{i m} g_{m i}}{h_{i i}} R-u_{m} R\right)}{\sum_{i} \frac{g_{i m}\left(I_{0}+\sigma_{i}^{2}\right)}{h_{i i}}+\sum_{i} \frac{g_{i m} g_{m i}}{h_{i i}} R\left(\sigma_{m}^{2}-\sum_{i} \frac{g_{i m}\left(I_{0}+\sigma_{i}^{2}\right)}{h_{i i}}\right)} .}
$$

For proof, the reader is referred to the Appendix B.

The value of $\lambda$ obtained by (22) must follow the condition (12) i.e.

$$
\lambda<\frac{h_{i i}}{g_{i m}\left(p_{m} g_{m i}+I_{i}+\sigma_{i}^{2}\right)}-\frac{u_{i}}{g_{i m}} .
$$

Let assume identical $g_{m i}, \quad \sigma_{i}^{2}$ and $I_{i}=0$ and arrange all FBSs in descending order as $\left(\frac{h_{11}}{g_{1 m} \sigma^{2}}-\frac{\mu_{1}}{g_{1 m}}>\frac{h_{22}}{g_{2 m} \sigma^{2}}-\frac{\mu_{2}}{g_{2 m}}>\ldots \ldots \ldots \frac{h_{N N}}{g_{N m} \sigma^{2}}-\frac{\mu_{N}}{g_{N m}}\right)$ then we can calculate the number of players that can participate in game by applying following condition

$$
\lambda<\left(\frac{h_{i i}}{g_{i m} \sigma^{2}}-\frac{\mu_{i}}{g_{i m}}\right)_{\min }
$$

The FBS for which the interference price does not fulfill the condition (24), its interference price is set to $\infty$ and it will be removed from the game.

When the co-tier interference changes, the new optimal interference price $\lambda^{*}$ can be determined in a similar manner as incorporated as described in Algorithm 1. Then, we can determine the optimal equilibrium power of FBSs and MBS by replacing the value of $\lambda^{*}$ in (19) and (20) respectively.
TABLE I

SYSTEM PARAMETERS

\begin{tabular}{ll}
\hline \hline Description & Values \\
\hline Number of FBSs & $3-15$ \\
Macrocell radius & $500 \mathrm{~m}$ \\
Femtocell radius & $15 \mathrm{~m}$ \\
Minimum inter-femto distance & $40 \mathrm{~m}$ \\
Minimum FBS-MUE distance & $20 \mathrm{~m}$ \\
Location of MUE or FUE & $\geq 0.7$ (cell radius) \\
$\mu_{m}$ & 0.01 \\
$\mu_{i}, \forall i$ & 0.01 \\
Outdoor path loss & $28+35 \log 10(d) \mathrm{dB}$ \\
Indoor path loss & $37+30 \log 10(d) \mathrm{dB}$ \\
Wall Penetration Loss (outdoor) & $10 \mathrm{~dB}$ \\
Wall Penetration Loss (indoor) & $5 \mathrm{~dB}$ \\
Shadowing standard deviation & $5 \mathrm{~dB}$ \\
for indoor \& outdoor links & \\
Thermal noise power & $-110 \mathrm{dBm}$ \\
Minimum MUE rate requirement & $0.5 \mathrm{bits} / \mathrm{sec} / \mathrm{Hz}$ \\
\hline \hline
\end{tabular}

\section{Simulation Results}

In this section, system level simulation is conducted to validate the performance of our power control scheme based on interference price. The detailed parameters of the channel model we use are given in Table 1. It is assumed that FBSs are concentrated near on the border of the MBS. And the performance of our scheme is also compared with an existing one in [10].

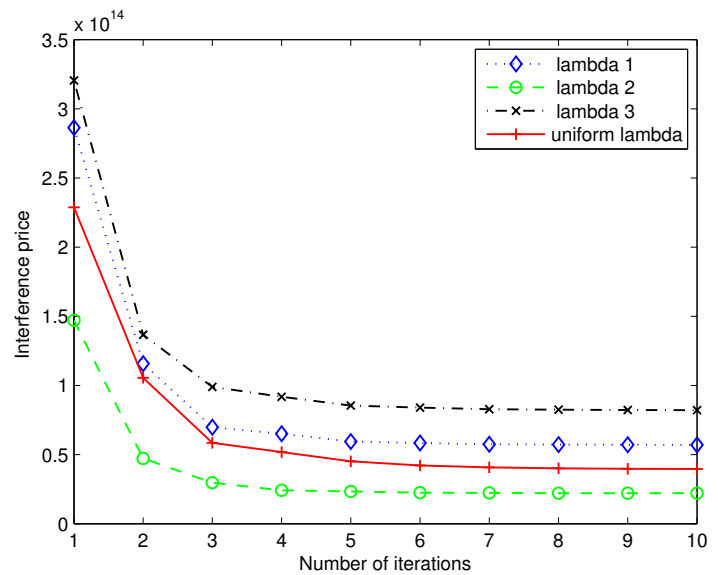

Fig. 1. Interference price vs number of iterations for 3 FBSs.

Firstly, we consider 3 FBSs randomly distributed near the MUE. Fig. 1 shows how the interference price changes with iteration while Fig. 2 shows the changes in MBS and FBSs power with iteration. Initially, MBS set high interference price by assuming FBSs co-tier interference $I_{i}=0$, thus FBSs transmit with low power. With calculation of $I_{i}, \mathrm{CC}$ starts lowering interference price and consequently MBS and FBSs power starts increasing. These observations are in accordance with the discussion made in section IV. It can be observed that price and power converge very rapidly after a few iterations which prove the convergence and efficiency of our algorithm. 


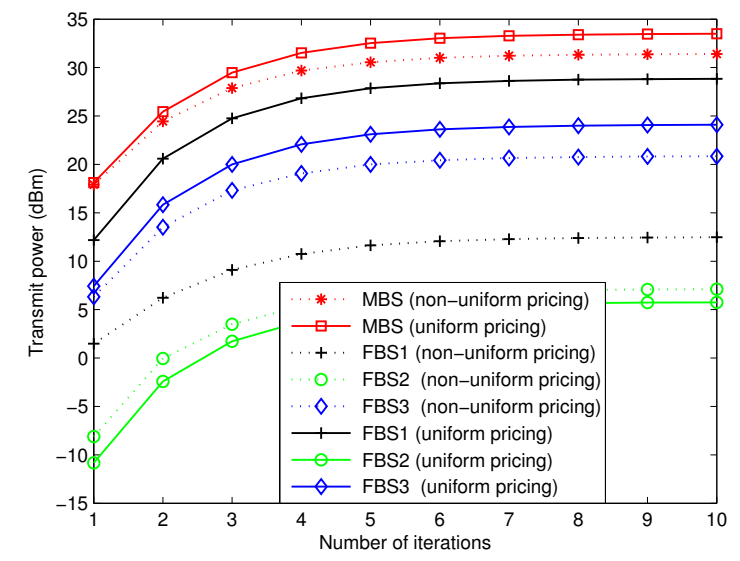

Fig. 2. MBS and 3 FBSs power allocation vs number of iterations.

The optimal transmitting power of the 3 FBSs is different, which can be explained by different interference price and channel gain from the FBSs to MUE.

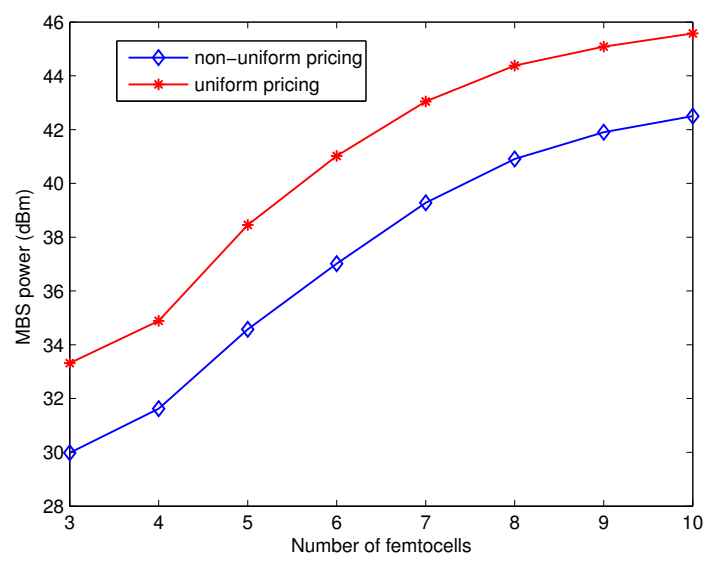

Fig. 3. MBS power allocation for non-uniform and uniform pricing.

Fig. 3 illustrates the relationship between MBS power and the number of transmitting FBSs in the network. When a new FBS is introduced into the network, higher interference is experienced by the MUE, thus MBS increases its power to maintain the QoS of its MUE. As the number of FBSs increases from 3 to 10, MBS power increases accordingly from $30 \mathrm{dBm}$ to $43 \mathrm{dBm}$ for non-uniform pricing and $33 \mathrm{dBm}$ to $45 \mathrm{dBm}$ for uniform pricing respectively. This means the results of our schemes are in line with analysis, which proves both schemes are reasonable. It also can be observed that uniform pricing scheme always set high MBS power than nonuniform pricing scheme with the same number of FBSs. We can conclude that deploying too many femtocells may not be appropriate, as the power of the MBS may be rise significantly due to high interference.

To evaluate the performance of our algorithm, an existing one described in [10] is chosen as comparison, where all

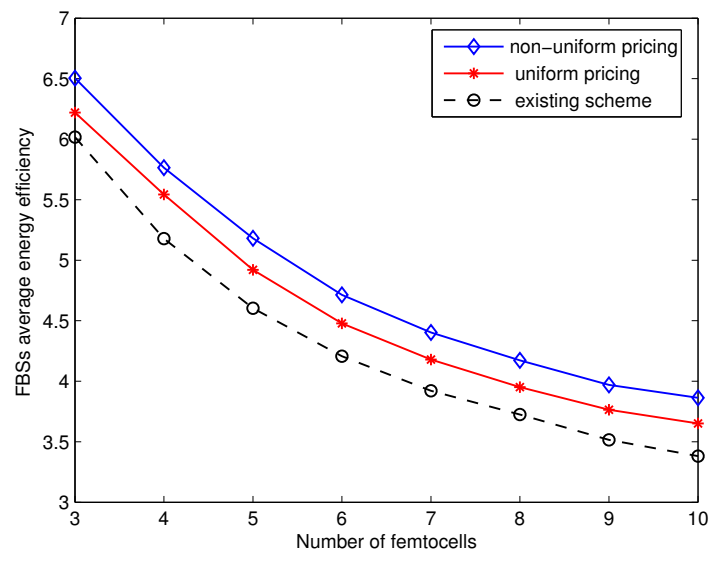

Fig. 4. Femtocells average energy efficiency.

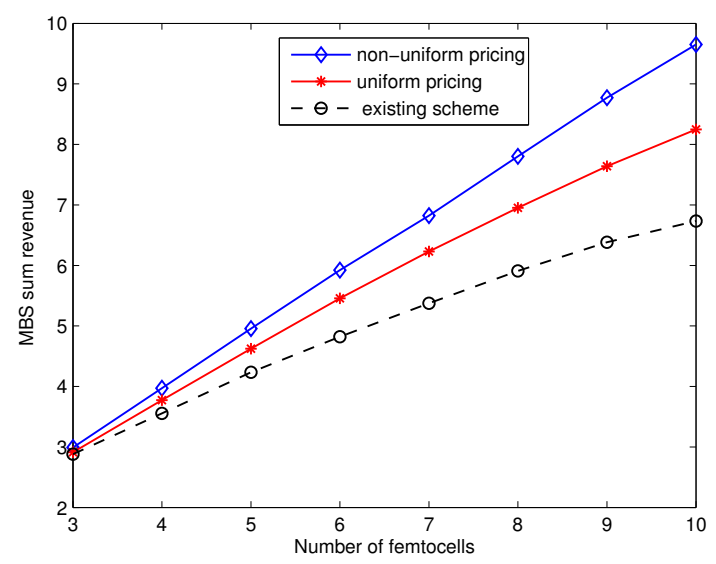

Fig. 5. MBS sum revenue.

derivations are made in terms of interference tolerance $Q$. We can rewrite from (3) and (6) with $Q=\frac{p_{m} h_{m m}}{e^{R^{\min }}-1}-\sigma_{m}^{2}$ and transform MBS power allocation given by our scheme to equivalent $Q$ values for the purpose of comparison. The results are shown in Fig. 4 and Fig. 5 in terms MBS sum revenue and average energy efficiency of FBSs. It can be observed that our algorithm provides enhanced performance in terms of both femtocell energy efficiency and MBS revenue over the existing one. This can be explained that by adding energy consumption in the utility function, the MBS and FBSs will not increase their power blindly to maximize their utility, which may happen for the comparison algorithm. It could also be observed that the performance of non-uniform interference price scheme is better than uniform interference price scheme. This is because the path-loss between the FBSs and MBS, thus different interference price should be used to show this difference in channel condition.

\section{CONCLUSIONS}

In this paper, we proposed an energy efficient interference management schemes for two-tier femtocell network, in which 
both energy consumption and spectrum efficiency are considered. The interference management problem is formulated using Stackelberg game. In the game, MBS acts as the leader which adjusts its power and imposes interference price on the FBSs to generate revenue. Based on this price, FBSs optimize their transmitting power to maximize their utility. In this way, the QoS of the MUE is guaranteed and energy efficiency is optimized. Two pricing strategies i.e. non-uniform and uniform pricing are introduced and the optimal solution under them is derived. We also proposed a low complexity iterative algorithm to calculate the interference price and power allocation strategy. The numerical results prove the enhanced performance of our proposed schemes over existing one in terms of both FBSs EE and MBS revenue.

\section{ACKNOWLEDGMENT}

This work was supported by the National Natural Science Foundation of China (61227801,61121001,61201152), the National Key Technology R\&D Program of China (2012ZX03003006), the National High Technology Research and Development Program of China (863 Program 2014AA01A707), the Program for New Century Excellent Talents in University (NCET-01-0259), and the Fundamental Research Funds for the Central Universities (2013RC0106).

\section{APPENDIX A}

\section{Proof of Proposition 4.1}

The objective function in Problem 4.1 is concave. Taking its derivative and setting $\frac{\partial U_{m}\left(\lambda_{i}\right)}{\partial \lambda_{i}}=0$, we can determine optimal value as

$$
\begin{gathered}
\frac{\partial U_{m}}{\partial \lambda_{i}}=\frac{g_{i m} \mu_{i}}{\left(\lambda_{i} g_{i m}+\mu_{i}\right)^{2}}-\frac{g_{i m}\left(I_{0}+\sigma_{i}^{2}\right)}{h_{i i}}-\frac{g_{i m}^{2} g_{m i} R \mu_{i}}{h_{i i}\left(\lambda_{i} g_{i m}+\mu_{i}\right)^{2}} \\
-\frac{g_{i m} g_{m i}}{h_{i i}} R\left(\sigma_{m}^{2}-\sum_{i} \frac{g_{i m}\left(I_{0}+\sigma_{i}^{2}\right)}{h_{i i}}\right)-\frac{\mu_{m} R g_{i m}^{2}}{\left(\lambda_{i} g_{i m}+\mu_{i}\right)^{2}}
\end{gathered}
$$

From the above we can get

$$
\lambda_{i}^{*}=\sqrt{\frac{\frac{\mu_{i}}{g_{i m}^{2}}-\frac{g_{m i}}{g_{i m} h_{i i}} R-\frac{\mu_{m} R}{g_{i m}}}{\frac{I_{0}+\sigma_{i}^{2}}{h_{i i}}+\frac{g_{m i}}{h_{i i}} R\left(\sigma_{m}^{2}-\sum_{i} \frac{g_{i m}\left(I_{0}+\sigma_{i}^{2}\right)}{h_{i i}}\right)}}-\frac{\mu_{i}}{g_{i m}}
$$

\section{APPENDIX B}

\section{Proof of Proposition 5.1}

The objective function in Problem 5.1 is concave. Taking its derivative and setting $\frac{\partial U_{m}(\lambda)}{\partial \lambda}=0$, we can determine optimal value as

$$
\begin{aligned}
\frac{\partial U_{m}}{\partial \lambda}= & \sum_{i} \frac{g_{i m} \mu_{i}}{\left(\lambda g_{i m}+\mu_{i}\right)^{2}}-\sum_{i} \frac{g_{i m} g_{m i} R}{h_{i i}} \sum_{i} \frac{g_{i m} \mu_{i}}{\left(\lambda g_{i m}+\mu_{i}\right)^{2}} \\
& -\sum_{i} \frac{g_{i m} g_{m i}}{h_{i i}} R\left(\sigma_{m}^{2}-\sum_{i} \frac{g_{i m}\left(I_{0}+\sigma_{i}^{2}\right)}{h_{i i}}\right) \\
& -\sum_{i} \frac{g_{i m}\left(I_{0}+\sigma_{i}^{2}\right)}{h_{i i}}-\mu_{m} R \sum_{i} \frac{g_{i m} \mu_{i}}{\left(\lambda g_{i m}+\mu_{i}\right)^{2}}
\end{aligned}
$$

To find the closed form solution we assume that $\lambda g_{i}$ is sufficient large such that $\lambda g_{i}+u_{i} \approx \lambda g_{i}$ then the above expression can be simplified as

$$
\begin{gathered}
\frac{\partial U_{m}}{\partial \lambda}=\frac{1}{\lambda^{2}} \sum_{i} \frac{\mu_{i}}{g_{i m}}-\frac{1}{\lambda^{2}} \sum_{i} \frac{g_{i m} g_{m i} R}{h_{i i}} \sum_{i} \frac{\mu_{i}}{g_{i m}} \\
-\sum_{i} \frac{g_{i m} g_{m i}}{h_{i i}} R\left(\sigma_{m}^{2}-\sum_{i} \frac{g_{i m}\left(I_{0}+\sigma_{i}^{2}\right)}{h_{i i}}\right) \\
-\sum_{i} \frac{g_{i m}\left(I_{0}+\sigma_{i}^{2}\right)}{h_{i i}}-\frac{\mu_{m} R}{\lambda^{2}} \sum_{i} \frac{\mu_{i}}{g_{i m}}
\end{gathered}
$$

From above we can write

$$
\lambda^{*}=\sqrt{\frac{\sum_{i} \frac{\mu_{i}}{g_{i m}}\left(1-\sum_{i} \frac{g_{i m} g_{m i}}{h_{i i}} R-\mu_{m} R\right)}{\sum_{i} \frac{g_{i m}\left(I_{0}+\sigma_{i}^{2}\right)}{h_{i i}}+\sum_{i} \frac{g_{i m} g_{m i}}{h_{i i}} R\left(\sigma_{m}^{2}-\sum_{i} \frac{g_{i m}\left(I_{0}+\sigma_{i}^{2}\right)}{h_{i i}}\right)}}
$$

\section{REFERENCES}

[1] I.W. Mustika, K. Yamamoto, H. Murata, and S. Yoshida "Potential game approach for self-organized interference management in closed access femtocell networks," IEEE VTC'11 (Spring), May 2011, pp. 1-5.

[2] D.T. Ngo, B.L. Long, LE-NGOC, and E. Hossain, "Distributed interference management in femtocell networks," IEEE VTC'11 (Fall), Sep. 2011, pp. 1-5.

[3] S. Guruacharya, D. Niyato, E. Hossain, and D.I. Kim, "Hierarchical competition in femtocell based cellular networks, "IEEE Globecom'10, Dec. 2010, pp. 1-5.

[4] X. Kang, R. Zhang, and M. Motani, "Price-based resource allocation for spectrum-sharing femtocell networks: A Stackelberg game approach," IEEE Journal on Sel.Areas in Commun., vol. 30, no. 3, pp. 538-549, April 2012.

[5] G. Miao, N. Himayat, G.Y. Li, and S. Talwar, "Distributed interferenceaware energy-effcient power optimization," IEEE Trans. on Wirel. Commun., vol. 10, no.4 pp. 1323-1333, April 2012.

[6] Renchao Xie, F.R. Yu, and Hong Ji "Spectrum sharing and resource allocation for energy-effcient heterogeneous cognitive radio networks with femtocells," IEEE ICC'11, June 2012, pp. 1661-1665.

[7] Tao Su, Wei Li, Wei Zheng, Xiangming Wen, and Zhen Liu "Energyefficient power optimization for two-tier femtocell networks using fictitious game," IEEE Globecom'12 , Dec. 2012, pp. 3478-3483.

[8] Youngwook Ko, and K. Moessner, "Maximum outage capacity in dense indoor femtocell networks with joint energy and spectrum utilization," IEEE Trans. on Wirel. Commun., vol. 11, no.12 pp. 4416-4425, Dec. 2012.

[9] Wei Li, Haijun Zhang, Wei Zheng, Tao Su and Xiangming Wen, "Energyefficient power allocation with dual-utility in two-tier OFDMA femtocell networks," IEEE GC Wkshps'12, Dec. 2012, pp. 535-540.

[10] X. Kang, Y.C. Liang and H.K. Garg, "Distributed power control for spectrum-sharing femtocell networks using Stackelberg game networks with femtocells," IEEE ICC'11, June 2011, pp. 1-5. 